\title{
Avoidance of psychological pain and suicidal ideation in community samples: Replication across two countries and two languages
}

\author{
Rui C. Campos ${ }^{1}$ (D) Ronald R. Holden ${ }^{2}$ (1) $\mid$ Christine E. Lambert ${ }^{2}$
}

${ }^{1}$ Departamento de Psicologia, Escola de Ciências Sociais e Centro de Investigação em Educação e Psicologia (CIEP-UE),

Universidade de Évora, Évora, Portugal

${ }^{2}$ Department of Psychology, Queen's University, Kingston, Ontario, Canada

\section{Correspondence}

Rui C. Campos, Departamento de Psicologia, Universidade de Évora, Apartado 94,

7002-554 Évora, Portugal.

Email: rcampos@uevora.pt

Funding information

Fundação para a Ciência e a Tecnologia, Grant/Award Number: UID/CED/04312/ 2019

\begin{abstract}
Objectives: A multidimensional psychological pain model of suicide has been developed that includes three dimensions: affective, cognitive, and avoidance. In the present research, we evaluated the relationships between the three dimensions of psychological pain and suicidal ideation.

Method: Two community samples from Portugal and the U.S. participated in the research.

Results: Hierarchical multiple regression analysis demonstrated that when controlling for the effects of depressive symptoms and psychache, the avoidance dimension, but not the cognitive or affective dimension of psychological pain, explained significant, and unique variance in suicidal ideation in each sample.

Conclusion: Even when individuals perceive psychological pain, there may not be an elevated risk of suicide, provided that they do not consider suicide as a form of coping to decrease that pain.

KEYWORDS

avoidance of pain, community samples, psychological pain, suicidal ideation
\end{abstract}

\section{1 | INTRODUCTION}

Suicidal ideation is an important risk factor for subsequent death by suicide (e.g., Baca-Garcia et al., 2011; Brezo et al., 2007). Several psychological variables (e.g., depression, hopelessness, and thwarted interpersonal needs) within the scope of various theoretical models of suicide (e.g., Interpersonal Theory of Suicide; Joiner, 2005) have been proposed as predisposing individuals toward suicidal ideation. One of the most compelling psychological 